Research Article

\title{
Improvement of Resveratrol Solubility by Complexation with Lactose Using Organic Solvent Spray Drying Technique
}

\author{
Bo Wang $\left(\mathbb{D}\right.$, Xinran Sun $\left(\mathbb{D}, X^{\prime}\right.$, Guo $\mathbb{D}$, Hanxi Yi, and Zeneng Cheng $(\mathbb{D}$ \\ Xiangya School of Pharmaceutical Sciences, Central South University, Changsha, Hunan 410000, China \\ Correspondence should be addressed to Zeneng Cheng; chengzn@csu.edu.cn
}

Received 12 August 2021; Accepted 7 September 2021; Published 6 October 2021

Academic Editor: tingting Hong

Copyright (C) 2021 Bo Wang et al. This is an open access article distributed under the Creative Commons Attribution License, which permits unrestricted use, distribution, and reproduction in any medium, provided the original work is properly cited.

\begin{abstract}
The purpose of this experiment is to change the crystallization state of resveratrol and improve its solubility and dissolution in water by spray drying technology, so as to improve the feasibility of resveratrol in clinical application. The powder samples were spray-dried with different proportions of ethanol aqueous solution as dissolution medium. The powder samples were characterized by infrared spectroscopy, thermogravimetry/differential scanning calorimetry, and ultraviolet spectroscopy, and their dissolution characteristics were investigated. It was found that the solubility and dissolution rate of different groups of samples obtained by spray drying increased compared with resveratrol crystals, and 50\% ethanol solution had the greatest solubilization effect. The experimental results show that this method has a positive effect on the solubility of resveratrol and can regulate its dissolution behavior.
\end{abstract}

\section{Introduction}

Resveratrol (3-4'-5-trihydroxystilbene) is a nonflavonoid polyphenolic compound with the chemical name $3,4^{\prime}, 5$-trihydroxy-1,2-diphenylethylene $\left(3,4^{\prime}, 5\right.$-stilbene), which is abundant in various fruits and their seeds, and is the main source of resveratrol in various berries $[1,2]$. Resveratrol was discovered as early as 1924 and was first isolated from the roots of Quercus furfuraceus in 1940. In 1976, resveratrol was also found in the leaves of grapes and is an antitoxin secreted by the plant body in response to adversity or pathogenic attack, with a dramatic increase in synthesis during UV exposure, mechanical damage, and fungal infection, hence the name phytoalexin $[2,3]$.

By forming hydrogen bonds and hydrophobic interactions with amino acid residues at the binding site, resveratrol and its analogs/derivatives are able to play a very outstanding positive role in the cardiovascular system and immune system through their hydroxyl groups that exhibit good interactions with target receptors [4-6]. These include (1) cardiovascular protection by reducing oxidative stress and inflammation, enhancing metabolic capacity, increasing nitric oxide synthe- sis, inhibiting VSMC proliferation, and enhancing autophagy [7-9]; (2) lowering fasting glucose for the treatment of diabetes [10]; and (3) even the alleviation and treatment of several cancers $[8,11,12]$. Despite the great potential of this compound for disease treatment, its clinical application has been limited by its low solubility and bioavailability [13].

The problem that researchers have been trying to solve is how to go about increasing the solubility of resveratrol in water and improving its in vivo bioavailability. In recent studies, several solutions have been proposed and these methods include lipid nanocarriers or liposomes, nanoemulsions, nanocrystals, etc. These methods do contribute to the solubility of resveratrol [14-17]. However, it is undeniable that nanomaterials or liposomes have disadvantages, such as difficult and unstable preparation, and excipients (i.e., ethanol and propylene glycol) which are known to have recognized actions or effects are usually used in the preparation [18]. Spray drying technology shows unique advantages because of its simple preparation and no special auxiliary materials.

As a process for the preparation of pharmaceutical particles, the spray drying technique allows the rapid drying of solutions or suspensions dispersed into small droplets to 


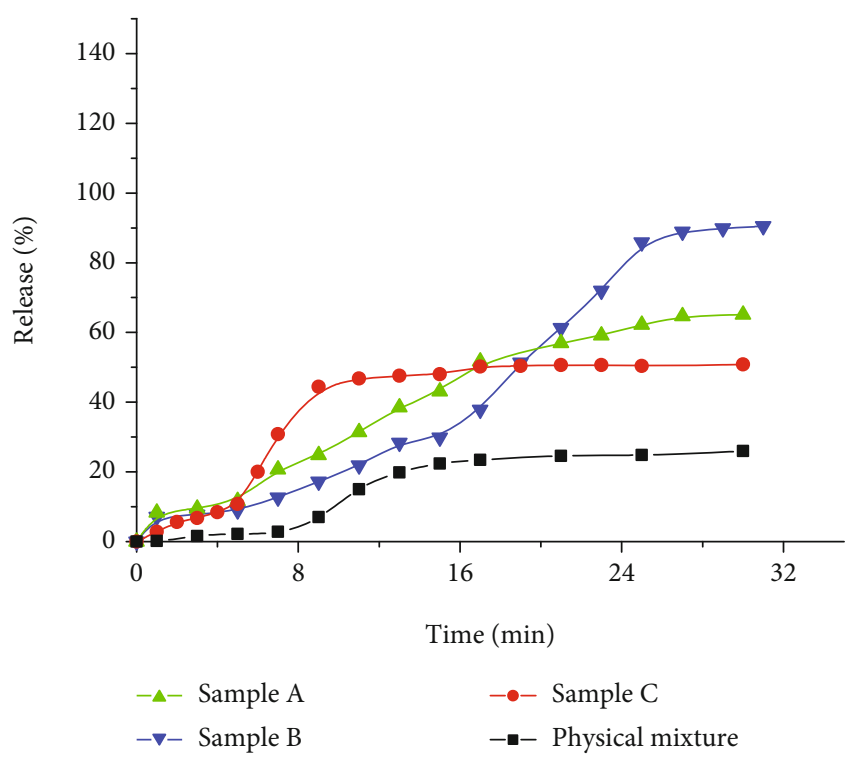

Figure 1: Dissolution curves of the three sample powders and physical mixture.

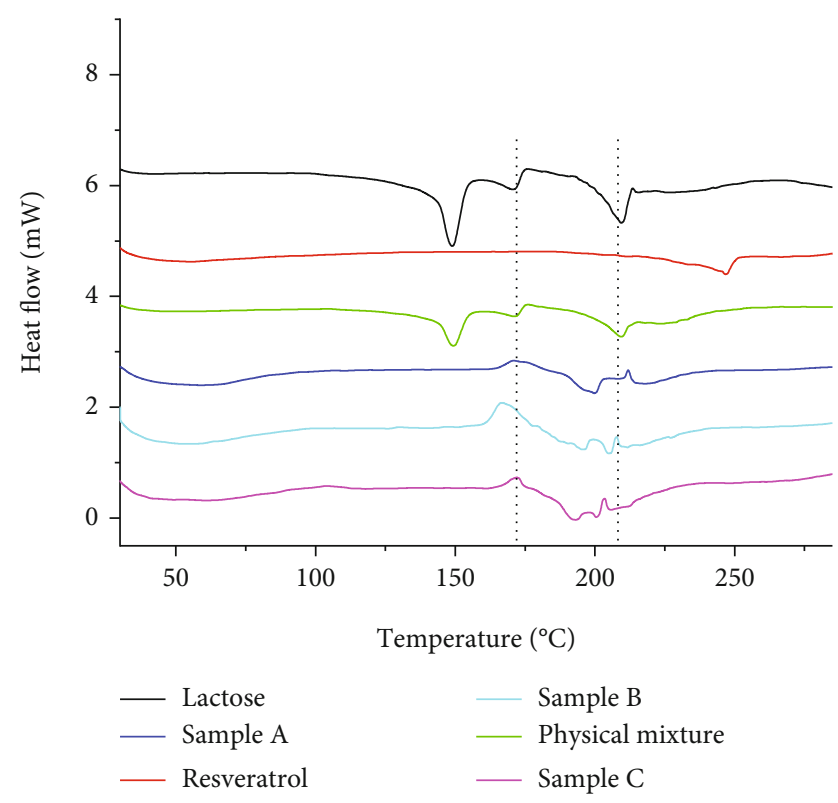

Figure 2: DSC curves of the raw materials and productions.

obtain a dry powder, and depending on the parameters, products with different properties can be obtained during the drying process [18]. When used to promote the solubility of insoluble drugs, spray drying techniques are commonly used to convert drugs to amorphous states or to prepare them as solid dispersions by cospraying with other excipients. In general, drugs in the amorphous state generally have a disordered lattice structure and generally have better solubility in water $[19,20]$.

In this experiment, the spray drying technology was used to mix the mixed solutions with different proportions (resveratrol in ethanol and lactose in water) according to the appropriate parameters, and the dispersion medium was

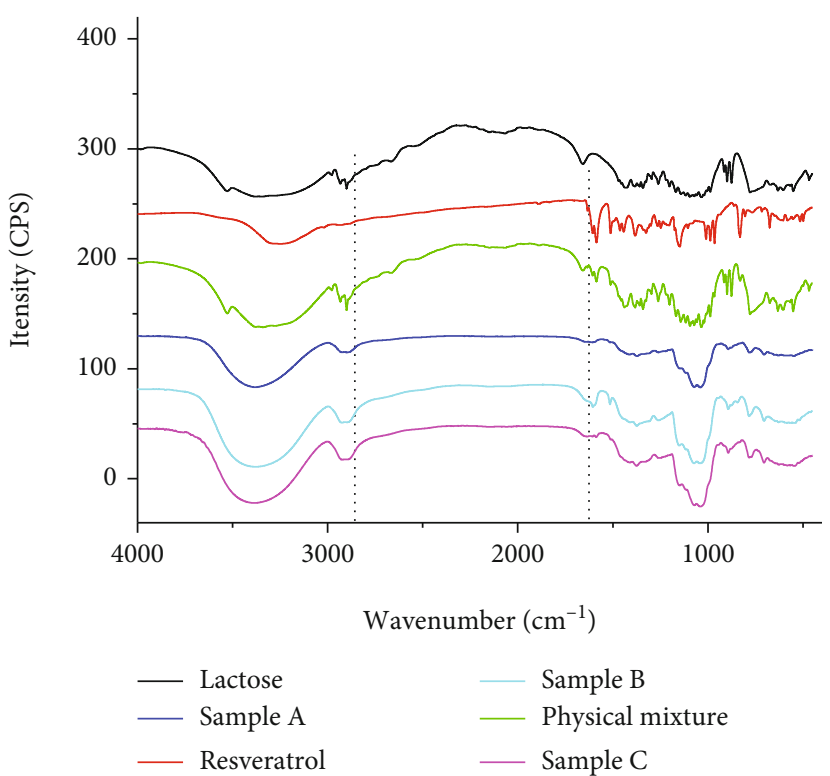

FIGURE 3: FTIR spectra of the raw materials and samples.

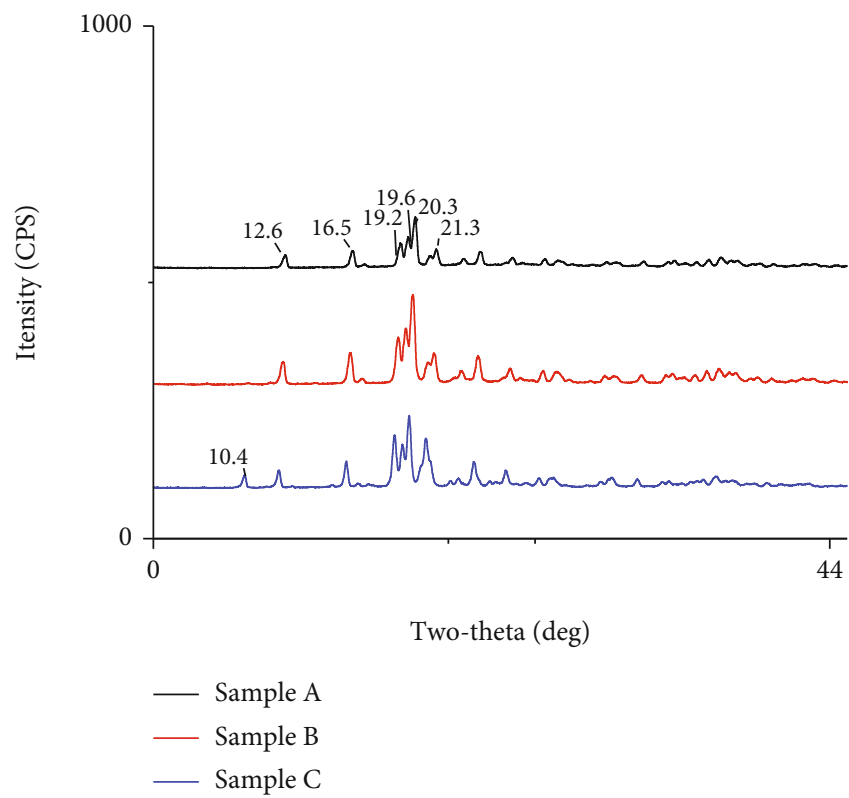

FIgURE 4: XRD curves of three samples.

removed to obtain the dried product. The dissolution characteristics and various physicochemical properties were characterized.

\section{Experimental Design}

In order to improve the solubility of low solubility resveratrol in water, we use spray dryer (Shanghai YC-015) to destroy the lattice structure of resveratrol and transform it into amorphous state, thus reducing the energy required for its dissolution in water. In order to improve the uniformity of mixing and increase the yield of products, we dried them together with lactose solution. It is reported that the 

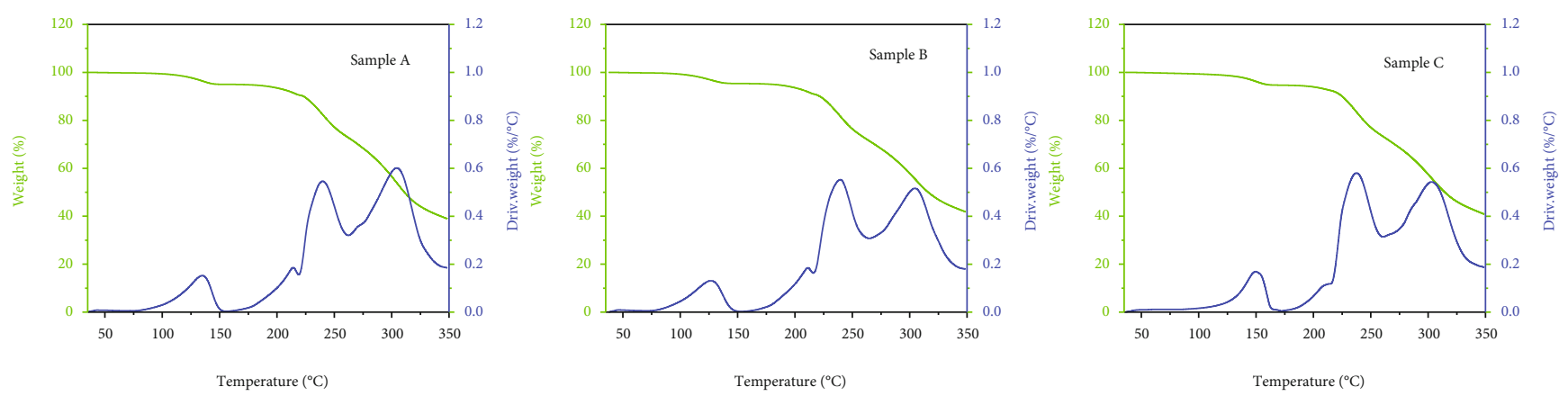

Figure 5: TGA curves of three samples (from left to right, samples A, B, and C).

solubility of resveratrol in ethanol is far greater than that in water. In order to dissolve resveratrol and increase the concentration of the solution as much as possible, we dissolved $1.0 \mathrm{~g}$ resveratrol in absolute ethanol (Shanghai Titan Technology Co., Ltd.) to prepare a 10\% lactose solution (Jiangsu Dawning Pharmaceutical Co., Ltd., China). After fully stirring and completely dissolving, the lactose solution is slowly added into the ethanol solution of resveratrol and stirred again to make it evenly dispersed. In this experiment, two parts of $1.0 \mathrm{~g}$ of resveratrol dissolved in $50 \mathrm{~mL}$ and $100 \mathrm{~mL}$ of absolute ethanol were accurately measured, and another $1.0 \mathrm{~g}$ of resveratrol was weighed and dispersed in pure water (manufactured in a laboratory) as a control. After fully stirring and completely dissolving, the lactose solution is slowly added to the ethanol solution of resveratrol and stirred again to make it evenly dispersed. The best parameters of spray drying are as follows: fan frequency $50.0 \mathrm{~Hz}$, inlet air temperature $140^{\circ} \mathrm{C}$, outlet a100ir temperature $1295^{\circ} \mathrm{C}$, peristaltic pump speed $10.0 \mathrm{~mL} / \mathrm{min}$, spray pressure $0.25 \mathrm{MPa}$, nozzle diameter $0.5 \mathrm{~mm}$, and three suspensions were spray dried to obtain samples A ( $33 \%$ ethanol solution $(v / v)$ ), B (50\% ethanol solution (50\% ethanol solution)), and C (100\% pure water solution).

\section{Characterization of Samples}

Samples A, B, C, and physical mixture were taken $0.35 \mathrm{~g}$ and compressed into tablets of $10 \mathrm{~mm}$ diameter under $10 \mathrm{MPa}$ pressure, and the dissolution data were obtained by RC1210G solubility meter (Xinzhi, China) with the extraction method of paddle method at $75 \mathrm{rpm} / \mathrm{min}, 37 \pm 0.3^{\circ} \mathrm{C}$, and sampling height of $750 \mathrm{~mL}$. Ultraviolet absorption spectra were obtained by Shimadzu, Japan. UV-dis2401 PC spectrophotometer, Shimadzu, Japan, was used to obtain UVvisible absorption spectra at $200-400 \mathrm{~nm}$. Raw materials and samples (RES crystals, lactose, and physical mixture as well as samples A, B, and C) were also studied using Fourier transform infrared spectroscopy (FTIR). Samples were compounded with dried $\mathrm{KBr}$ powder, pressed into transparent sheets, and scanned for transmission sensitivity on a Nicolet 6700 FTIR spectrometer (Thermo Fisher Scientific). The resolution of FTIR spectra was $1 \mathrm{~cm}^{-1}$. The microscopic appearance of the samples was examined with a JSM-7200F scanning electron microscope (SEM, JEOL Ltd.). Thermal analysis was also performed using DSC and TGA to analyze

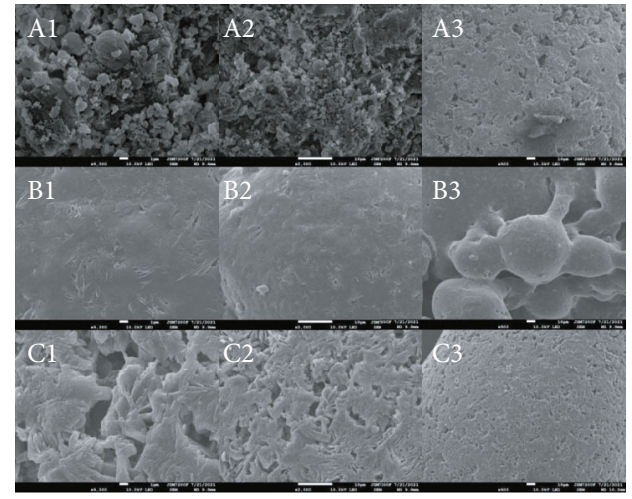

Figure 6: SEM images of sample A (A1,A2, andA3), sample B (B1, $\mathrm{B} 2$, and $\mathrm{B} 3)$, and sample $\mathrm{C}(\mathrm{C} 1, \mathrm{C} 2$, and $\mathrm{C} 3)$, respectively.

the structural features. The model number of the differential scanning calorimeter was HSC-4 DSC, Hengjiu, China. The samples used for DSC determination were prepared in sealed aluminum pans according to standard procedures. Approximately $5.0 \mathrm{mg}$ of each specimen was used for the analysis. The samples were heated from $30^{\circ} \mathrm{C}$ to $300^{\circ} \mathrm{C}$ at a rate of $5^{\circ} \mathrm{C} / \mathrm{min}$. $\mathrm{N}_{2}$ was used as purge gas to obtain the heat flow versus temperature rise in the temperature range of 30 $300^{\circ} \mathrm{C}$. The specific parameters of the thermogravimetric analyzer (TGA Q5000 V3.17 Build 265) used for the thermogravimetric analysis were temperature: $35^{\circ} \mathrm{C} 350^{\circ} \mathrm{C}$, heating rate $5^{\circ} \mathrm{C} / \mathrm{min}$, samples were tested in an alumina pot, and $\mathrm{N}_{2}$ was used as the equilibrium gas. The crystallization characteristics of the three sample specimen powders were studied by XRD analysis. The solid samples were scanned with a diffraction angle $(2 \theta)$ of $5 \sim 40^{\circ}$ using a Siemens D5000 diffractometer. The sample mass in the powder box was $0.5 \mathrm{~g}$. The scanning rate was $1.2^{\circ} / \mathrm{min}$, the scanning current was $30 \mathrm{~mA}$, and the scanning voltage was $40 \mathrm{kV}$. We also performed $\mathrm{N}_{2}$ adsorption experiments on the samples separately to evaluate their relative pore size and ratio.

\section{Results and Discussion}

We measured the dissolution and release characteristics of three sample powders and physical mixtures and plotted their dissolution curves, as shown in Figure 1. Compared with the physical mixture, the solubility of all three spray- 

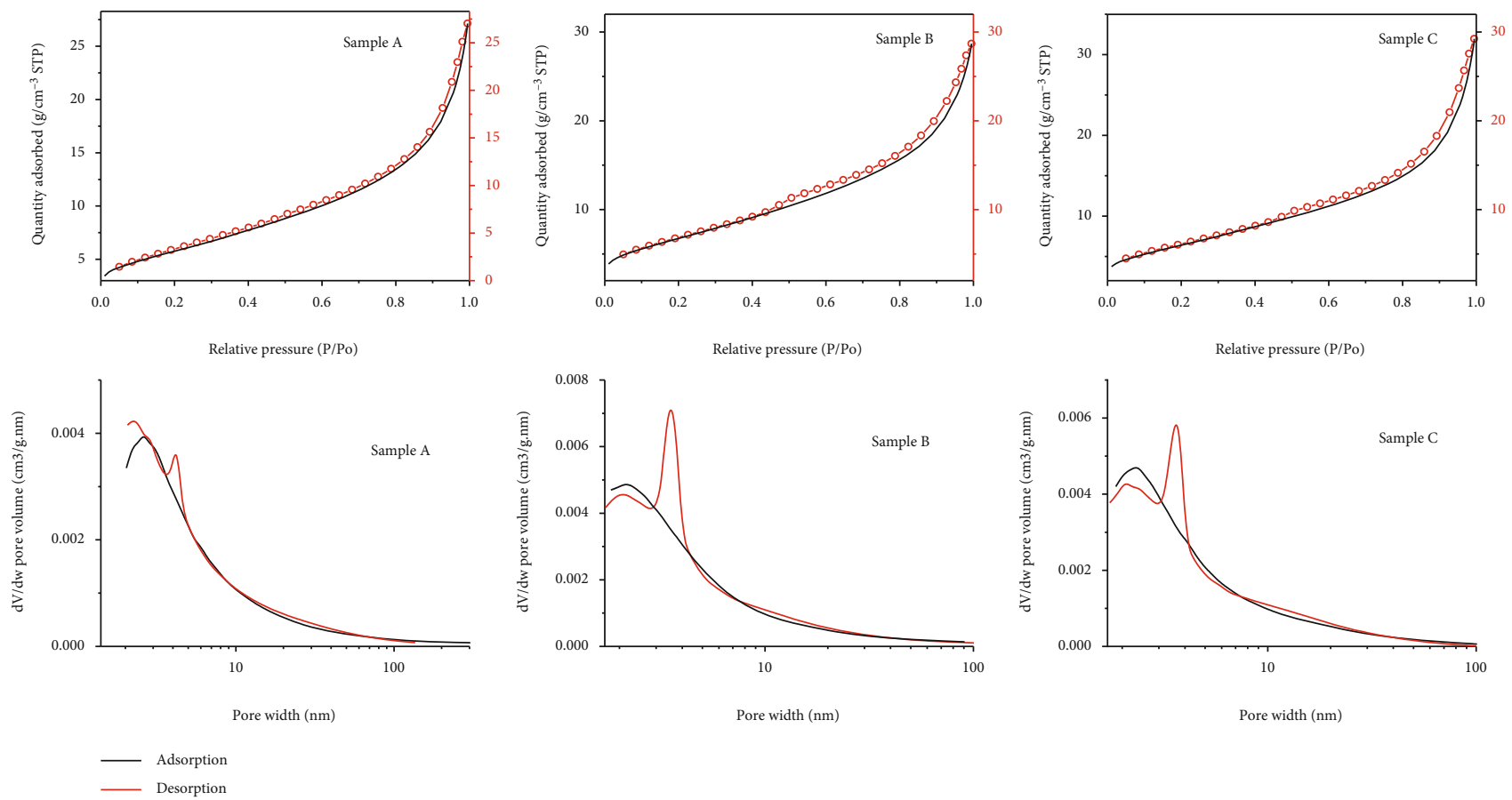

Figure 7: BET (upper) and $\mathrm{BJH}$ (lower) curves of samples $\mathrm{A}, \mathrm{B}$, and $\mathrm{C}$ (from left to right).

dried samples increased, and sample B (50\% ethanol aqueous solution) was the most obvious. It can be seen from the figure that the solubility and solubility of the physical mixture are the lowest. With the increase of ethanol dosage, its solubility increases, but the dissolution rate of predissolution decreases. The analysis may be because the larger the volume of ethanol, the more the lattice structure of resveratrol is destroyed, so the proportion of amorphous resveratrol in the product increases, resulting in an increase in dissolution. The increase of ethanol ratio leads to the precipitation of more lactose from the mixed solution, which reduces the amorphous lactose in the product and slows down the disintegration of the drug, resulting in the decrease of dissolution rate. Infrared spectroscopy and differential scanning calorimetry also confirmed it.

The DSC curves of several starting materials and samples are shown in Figure 2. The vaporization peak of the water of crystallization and the endotherm of the lactose crystal melting occur at 148.9 and $209.5^{\circ} \mathrm{C}$, respectively, and the exothermic crystallization peak of amorphous lactose occurs at $176.0^{\circ} \mathrm{C}$. The endothermic peak API for resveratrol occurs at $240-260^{\circ} \mathrm{C}$. The resveratrol peak overlapped with the melting peak of the lactose crystals in the physical mixture, and the other peaks of lactose existed separately and in the same position. For spray-dried samples, amorphous peaks exhibited varying degrees of increase and shift relative to the physical mixture, with changes in amorphous content and composition expected. Among the three samples, the amorphous peak in sample $\mathrm{C}$ was the narrowest, and that in sample B was the widest, with more shifts to the lowtemperature region. As can be seen, sample B formed a complex coamorphous structure while the amorphous form of sample $\mathrm{C}$ was a single component. Among the three groups of samples, the crystalline peak of resveratrol in group B was the smallest, and the amorphous peak was the largest, with a narrow peak width. The resveratrol crystal peak in group $C$ was the largest, and it moved to the low-temperature region relative to other groups. Based on the comprehensive analysis, it could be concluded that the larger the proportion of ethanol was, the greater the dissolution rate of resveratrol in the mixture would be. However, at the same time, more lactose crystals would be separated out during the spray drying process, which reduced the amorphous lactose in the product and led to the slow dissolution rate.

The variation of the intensity or wave number of the peaks was studied by FTIR spectroscopy (Figure 3). The methylene vibrational peaks of lactose at $3000-2950 \mathrm{~cm}^{-1}$ and the complex spikes of resveratrol at $1600-1000 \mathrm{~cm}^{-1}$ became milder relative to the raw material and the physical mixture. This indicates a decrease in the density and strength of hydrogen bonds, a decrease in the crystalline state, a decrease in the level of intramolecular interactions, a dispersion of vibrational energy levels, and a decrease in the conceptual selectivity [21, 22]. This indicates that the amorphous content is higher in each sample. Sample B shows a similar vibrational peak at $1600 \mathrm{~cm}^{-1}$ to the raw lactose material, indicating a higher lactose crystal content than the other samples.

Based on the XRD curve (Figure 4), the $\alpha$-lactose peak was $12.6^{\circ}, 19.2^{\circ}, 19.6^{\circ}$, and $20.3^{\circ}$ for the three samples at $2 \theta$, and the $\beta$-lactose peak was 10.4 for sample $\mathrm{C}$ at $2 \theta$, but not for samples $A$ and $B$. This indicated that in the ethanol-free solution, the free state of lactose exhibited variable rotational behavior, which was inhibited at low ethanol concentrations. Spray drying gave the least amount of amorphous lactose and the most amorphous resveratrol at high ethanol concentrations, with sharp and steep lactose peaks, while different amounts of amorphous lactose product were detected in other samples. 
According to the TGA (Figure 5) curves, the weight loss trends of the three groups of samples were basically the same in the same temperature range, and the thermal stability was also basically similar and identical. Throughout the warming process, the samples were roughly divided into four steps of weight loss, and the first step of weight loss showed that water was present in all three samples and the water content was basically the same. The second weight loss peak was at $200-250^{\circ} \mathrm{C}$, which, when combined with DSC analysis, indicated that sample $B$ probably produced the most amorphous resveratrol and sample $C$ the least, which is consistent with the data obtained from the dissolution curve analysis.

From the SEM images (Figure 6) and the comprehensive analysis of the $\mathrm{BET}$ and $\mathrm{BJH}$ data (Figure 7), we found that there were pore-like structures on the surface and inside of each sample, and there was no hysteresis loop for the adsorption isotherm. The consistent type of the isotherm indicated that the same type of pores existed in each sample. The average pore diameters of samples $\mathrm{A}$, $\mathrm{B}$, and $\mathrm{C}$ were in the range of $7.2-8.0 \mathrm{~nm}$, where the minimum average pore diameter of sample $B$ was $7.25 \mathrm{~nm}$, the maximum pore surface area was $24.45 \mathrm{~m}^{2} / \mathrm{g}$, and the larger pore volume was $0.044 \mathrm{~cm}^{3} / \mathrm{g}$, indicating that under the same spray-drying parameters, the appropriate proportion of ethanol was conducive to the pore-forming of particulate matter, more conducive to the loading of drugs, and increasing the solubility.

\section{Conclusion}

In this experiment, we obtained mixed solutions with different ethanol ratios. Different samples were obtained by spray drying technology, and the solvent was instantly dried. By measuring their release curves in vitro, we found that the solubility of samples obtained by spray drying increased in all cases, but the solubilization rate was different according to the composition of solvents. The solubilization characteristics of lactose and resveratrol are different in different solvents. After spray drying, the two substances will produce amorphous products with different proportions. The results showed that after spray drying, the solubility of resveratrol increased, and the solubilization effect was more obvious for the solution samples with high ethanol content, but the early release rate decreased. After spray drying, different proportions of ethanol lead to different crystallization behaviors, but all samples have high porosity. In a word, cospray drying technology can effectively increase the solubility of resveratrol, and the solubilization effect and dissolution rate vary with the solvent composition. We can use this method to effectively improve the solubility and adjust the dissolution behavior of insoluble drugs.

\section{Data Availability}

All data used to support the findings of this study are included within the article.

\section{Conflicts of Interest}

We declare that we have no financial and personal relationships with other people or organizations that can inappropriately influence our work.

\section{References}

[1] C.-Y. Hou, Y.-L. Tain, H.-R. Yu, and L.-T. Huang, "The effects of resveratrol in the treatment of metabolic syndrome," International Journal of Molecular Sciences, vol. 20, no. 3, p. 535, 2019.

[2] M. Berretta, A. Bignucolo, R. Di Francia et al., "Resveratrol in cancer patients: from bench to bedside," International Journal of Molecular Sciences, vol. 21, no. 8, p. 2945, 2020.

[3] B. Tian and J. Liu, "Resveratrol: a review of plant sources, synthesis, stability, modification and food application," Journal of the Science of Food and Agriculture, vol. 100, no. 4, pp. 1392$1404,2020$.

[4] Y. Shi, J. Zhou, B. Jiang, and M. Miao, "Resveratrol and inflammatory bowel disease," Annals of the New York Academy of Sciences, vol. 1403, no. 1, pp. 38-47, 2017.

[5] Y. J. Song, C. B. Zhong, and W. Wu, "Resveratrol and diabetic cardiomyopathy: focusing on the protective signaling mechanisms," Oxidative Medicine and Cellular Longevity, vol. 2020, Article ID 7051845, 19 pages, 2020.

[6] P. Silva, A. Sureda, J. A. Tur, P. Andreoletti, M. CherkaouiMalki, and N. Latruffe, "How efficient is resveratrol as an antioxidant of the Mediterranean diet, towards alterations during the aging process?" Free Radical Research, vol. 53, no. sup1, pp. 1101-1112, 2019.

[7] X. Li and D. Hasselwander, "Resveratrol and vascular function," International Journal of Molecular Sciences, vol. 20, no. 9, p. 2155, 2019.

[8] S. Galiniak, D. Aebisher, and D. Bartusik-Aebisher, "Health benefits of resveratrol administration," Acta Biochimica Polonica, vol. 66, no. 1, pp. 13-21, 2019.

[9] J. M. Breuss, A. G. Atanasov, and P. Uhrin, "Resveratrol and its effects on the vascular system," International Journal of Molecular Sciences, vol. 20, no. 7, p. 1523, 2019.

[10] M. Springer and S. Moco, "Resveratrol and its human metabolites-effects on metabolic health and obesity," Nutrients, vol. 11, no. 1, p. 143, 2019.

[11] R. Kataria and A. Khatkar, "Resveratrol in various pockets: a review," Current Topics in Medicinal Chemistry, vol. 19, no. 2, pp. 116-122, 2019.

[12] T. Kisková and M. Kassayová, "Resveratrol action on lipid metabolism in cancer," International Journal of Molecular Sciences, vol. 20, no. 11, p. 2704, 2019.

[13] L. Malaguarnera, "Influence of resveratrol on the immune response," Nutrients, vol. 11, no. 5, p. 946, 2019.

[14] A. Chimento, F. De Amicis, R. Sirianni et al., "Progress to improve oral bioavailability and beneficial effects of resveratrol," International Journal of Molecular Sciences, vol. 20, no. 6, p. 1381, 2019.

[15] L. Montenegro, C. Parenti, R. Turnaturi, and L. Pasquinucci, "Resveratrol-loaded lipid nanocarriers: correlation between in vitro occlusion factor and in vivo skin hydrating effect," Pharmaceutics, vol. 9, no. 4, p. 58, 2017.

[16] G. Davidov-Pardo and D. J. McClements, "Nutraceutical delivery systems: resveratrol encapsulation in grape seed oil 
nanoemulsions formed by spontaneous emulsification," Food Chemistry, vol. 167, pp. 205-212, 2015.

[17] B. M. Steiner, V. Shukla, D. J. McClements, Y. O. Li, M. Sancho-Madriz, and G. Davidov-Pardo, "Encapsulation of lutein in nanoemulsions stabilized by resveratrol and Maillard conjugates," Journal of Food Science, vol. 84, no. 9, pp. 2421-2431, 2019.

[18] A. Ziaee, A. B. Albadarin, L. Padrela, T. Femmer, E. O'Reilly, and G. Walker, "Spray drying of pharmaceuticals and biopharmaceuticals: critical parameters and experimental process optimization approaches," European Journal of Pharmaceutical Sciences, vol. 127, pp. 300-318, 2019.

[19] A. H. Salama, "Spray drying as an advantageous strategy for enhancing pharmaceuticals bioavailability," Drug Delivery and Translational Research, vol. 10, no. 1, pp. 1-12, 2020.

[20] K. B. Shepard, A. M. Dower, A. M. Ekdahl, M. M. Morgen, J. M. Baumann, and D. T. Vodak, "Solvent-assisted secondary drying of spray-dried polymers," Pharmaceutical Research, vol. 37, no. 8, p. 156, 2020.

[21] Z. Hajihashemi, A. Nasirpour, J. Scher, and S. Desobry, "Interactions among lactose, $\beta$-lactoglobulin and starch in colyophilized mixtures as determined by Fourier transform infrared spectroscopy," Journal of Food Science and Technology, vol. 51, no. 11, pp. 3376-3382, 2014.

[22] M. A. Ottenhof, W. MacNaughtan, and I. A. Farhat, "FTIR study of state and phase transitions of low moisture sucrose and lactose," Carbohydrate Research, vol. 338, no. 21, pp. 2195-2202, 2003. 